\title{
Soporte Para la Generación de Maquetas Virtuales de Automatización Industrial
}

\author{
A. Sánchez, E. Estévez, I. Ruano Ruano, Juan Gómez Ortega, Javier Gámez García \\ Escuela Politécnica Superior de Jaén. \\ Universidad de Jaén \\ Email: \{asgarcia,eestevez, alonso,juango,jggarcia \}@ujaen.es
}

\begin{abstract}
Resumen
Está demostrada la importancia que tienen la realización de prácticas de laboratorio en la educación universitaria relacionada con las ciencias, tecnologías, ingenierías y matemáticas. En el caso de las ingenierías y en particular en conceptos de automatización se hace aún más notorio. Lamentablemente, situaciones como las vividas en estos dos últimos cursos académicos, en los que la docencia presencial no ha sido posible, ha puesto a prueba tanto a docentes como a alumnos. Este trabajo presenta unas directrices y una herramienta de soporte para que el profesorado de este tipo de asignaturas pueda generar maquetas virtuales y por tanto realizar las prácticas de forma online de la misma manera que presencialmente.
\end{abstract}

Palabras Clave: Docencia Virtual, Automatización industrial.

\section{INTRODUCCIÓN}

Las prácticas de laboratorio constituyen un recurso docente muy importante en la docencia universitaria [1]. En los estudios universitarios de ciencias y tecnologías la importancia de los laboratorios es aún mayor, ya que sus actividades suelen ser eminentemente prácticas [2]-[3]. De hecho, el trabajo de laboratorio ayuda a los alumnos a entender la realidad a la que se deben enfrentar, estableciendo un nexo de unión entre el mundo teórico docente y el mundo real.

Algunos autores han llegado a afirmar que las experiencias de laboratorio constituyen el corazón del aprendizaje científico [4], o que las experiencias de laboratorio hacen que la ciencia "cobre vida" [5]. El trabajo práctico constituye un pilar esencial en la formación de los estudiantes de ingeniería, que deben tener un conocimiento que vaya más allá de la propia teoría [2]. La comunidad científica presenta un acuerdo explícito sobre los beneficios que aportan los laboratorios en la enseñanza de ciencias e ingenierías [3],[6]-[9].
Por otro lado, los profesionales de ingeniería llevan mucho tiempo siendo artífices de muchos cambios que se han producido gracias a la explotación de avances tecnológicos y sus innovaciones relacionadas [10]. La aplicación de estos avances tecnológicos en la educación, especialmente de las Tecnologías de la Información y las Comunicaciones (TIC), que posibilitan la formación online, es una línea de actuación muy importante que fomentan ampliamente organismos públicos y privados, tanto internacionalmente [11]-[12] como en España [13].

El avance y la aplicación de las TIC ha traído consigo la aparición de nuevos tipos de laboratorios con los que los alumnos pueden trabajar a través de Internet en lo que se ha venido a llamar laboratorios online (LoL) o en línea [14]. Es más, en la literatura se pueden encontrar muchas propuestas de laboratorios online muy útiles para la disciplina de ISA (Ingeniería de Sistemas y Automática) [15]-[21] siendo la mayoría de ellos para temas de fundamentos de control.

Lamentablemente, en la temática de Automatización industrial, al menos hasta donde los autores conocen, no se dispone de dicha batería de laboratorios. Fundamentalmente se debe a que para esta materia se requiere de herramientas propietarias de fabricantes (e.g. TIA Portal para autómatas de Siemens, Twincat para Beckhoff...) que dan soporte a la codificación de los proyectos de automatización y posteriormente descargarlos en el correspondiente Autómata Programable (PLC - Programmable Logic Controller) o en una herramienta que simule el PLC (también propietaria, e.g., PLCsim de Siemens). Hoy en día, para impartir asignaturas de automatización industrial, los centros disponen de laboratorios dotados al menos de PLCs, manipuladores de dos grados de libertad (i.e., cilindros de simple o doble efecto gobernados por correspondientes electroválvulas) ...lo cual permite asegurar una docencia de calidad, aunque de recursos limitados y compartidos por todo el alumnado. 
Estos dos últimos años donde la situación de alarma que se ha vivido en la sociedad debida al COVID-19 donde la docencia presencial no ha sido posible [22], ha dificultado muy mucho la impartición de dichas asignaturas. En el mejor de los casos; suponiendo que el alumnado tenía recursos suficientes y conexión a red y la universidad ofrecía conexión a PC remotos con licencias para codificar el proyecto de automatización; se ha podido diseñar y codificar los ejercicios propuestos. Aun así, no se tenía disponible ni planta real ni virtual a automatizar y controlar. Ello, ha dificultado muy notoriamente la comprensión del alumnado.

En el mercado, se dispone de productos comerciales como Factory I/O [23]o Fluidsim [24] que podrían ser utilizados para emular la planta a automatizar y posteriormente establecer la conexión con el PLC (real o virtual). El software Factory IO permite una perfecta simulación de los sistemas de transporte, distribución y almacenaje de paquetería en entornos industriales, pero no permite añadir nuevos objetos, muy utilizados en asignaturas básicas de automatización como pueden ser cilindros o motores. Fluidsim permite añadir dichos manipuladores, pero con una apariencia muy poco real. Además, hay que tener en cuenta el coste sus licencias.

Este trabajo propone una herramienta que da soporte a la generación de maquetas virtuales para prácticas de Automatización Industrial. Dicha herramienta está basada en librerías de libre distribución y permite al docente elaborar plantas de complejidad creciente que sirvan de prácticas de automatización para el alumnado.

El resto del artículo está organizado de la siguiente manera: el apartado 2 lista las pautas para conseguir la virtualización de maquetas de automatización; el apartado 3 presenta la herramienta desarrollada que guía a la implementación de las pautas previamente indicadas. El apartado 4 hace uso de la herramienta propuesta para la virtualización de una maqueta utilizada en la asignatura de Automática Industrial impartida en los Grados de Ingeniería Industrial de la Escuela Politécnica Superior de Ingeniería (EPSj) de Universidad de Jaén (UJA). Finalmente, el apartado 5 recoge las conclusiones.

PAUTAS PARA
VIRTUALIZACIÓN
PRÁCTICAS
AUTOMATIZACIÓN

La virtualización de maquetas de automatización está directamente ligada con un concepto de gran repercusión en el contexto de la industria 4.0 como es el Gemelo Digital (DT-Digital Twin). Hoy en día, no hay un consenso en cuanto a las funcionalidades y alcance que debe tener un DT, es más, en la literatura se han dado tres interpretaciones: (1) modelo de simulación de alta precisión [25]-[26]; (2) representación virtual del estado y comportamiento de un activo de fabricación para su integración en la I4.0 [27]-[28] y (3) una combinación de las dos primeras [29]-[30].

Este trabajo, concretamente está en concordancia con la primera de las interpretaciones de DT, ya que con la maqueta virtual se pretende obtener un modelo de simulación de alta precisión para el análisis y optimización de proceso de fabricación. Las interfaces de $\mathrm{E} / \mathrm{S}$ de la maqueta virtual son las mismas que las del proceso que replica/virtualiza, así para el controlador la interacción con el proceso real y con su virtualización es la misma.

Las acciones para desarrollar un gemelo digital de estas características son las siguientes:

1. Modelado de la planta a automatizar. El primer paso consiste en la generación del modelo de simulación de la planta a automatizar.

2. Proyecto de automatización. Destacar que la lógica de dicho proyecto ha de ser válida tanto para el DT como para el activo de fabricación real (i.e., Maqueta real en nuestro caso).

3. Integración en arquitecturas para la Industria 4.0. Este paso, consiste dotar al proyecto de automatización de recursos para procesar los datos que recibe del proceso (real o el simulado).

\section{HERRAMIENTA SOPORTE DE VIRTUALIZACIÓN}

Este apartado presenta una herramienta de soporte que permita al profesorado generar DT para fomentar las prácticas de automatización, de tal manera que no requiera de licencias adicionales. En concreto está basada en software abierto y de libre distribución.

La Figura 1 presenta un escenario general haciendo énfasis en las librerías empleadas. En la actualidad da soporte para automatizar y controlar plantas virtuales cuyo proyecto de automatización se haya desarrollado en dos de las herramientas más extendidas en el mundo de la automatización como son TIA Portal (Siemens) y Twincat (Beckhoff).

Los siguientes apartados detallan cómo permite modelar la planta a controlar, definir las E/S que van a intercambiarse con el proyecto de automatización, así como su integración y puesta en marcha. 


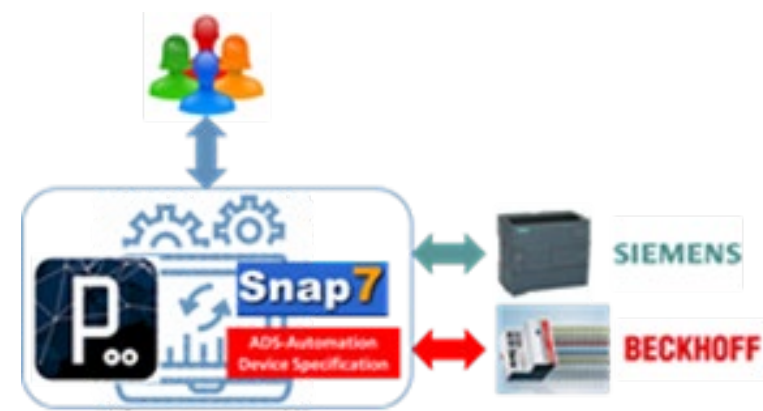

Figura 1: Escenario general de la herramienta propuesta

\subsection{Modelado de la planta a automatizar $y$ controlar}

El Modelado de la planta a automatizar, requiere de un CAD por cada módulo que la compone. En caso de tratarse de un módulo con movimiento (e.g., un cilindro), son necesarios dos $\mathrm{CAD}$, uno para la parte fija (estructura) y otro para la parte móvil (émbolo).

El núcleo de la herramienta maneja un fichero de configuración en formato yaml [31] para cada elemento de la maqueta y otro general donde se hace la composición de la misma. De esta manera, se consigue una mayor flexibilidad. La Figura 2 presenta un ejemplo de fichero de configuración de dos elementos clásicos en cualquier planta como son los cilindro y pulsadores. En el caso de los cilindros además de su ubicación, es necesario indicar la longitud del émbolo (carreraMax, carreraMin), la velocidad de movimiento, el gráfico de la parte fija y móvil (ficheroFijo, ficheroMovil) y los correspondientes finales de carrera (indicadorl, indicador2).

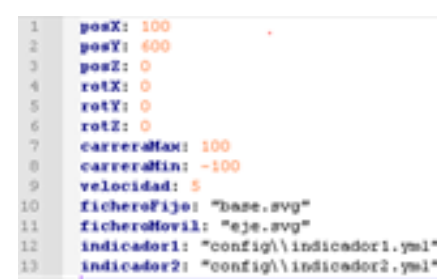

(a) Cilindro

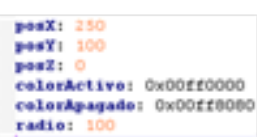

(b) Pulsador
Figura 2: Ejemplos de parametrización: (a) cilindro y (b) pulsador.

\subsection{Proyecto de Automatización}

Tal y como ilustra la Figura 1 la herramienta se ha de encargar de comunicar la maqueta con el proyecto de automatización que se estará ejecutando en un real o virtual. Por tanto, la herramienta es responsable de consultar las salidas y actualizar las entradas del controlador. Para ello, se ha de conocer las direcciones de E/S con las que se trabaja, así como la dirección IP del controlador.
Si el proyecto de automatización se ejecuta en un PLC real o virtual de Siemens (PLCSim):

a. Hace falta una configuración específica del proyecto de automatización codificado en TIA Portal para dejar que desde el exterior se pueda acceder a la memoria de E/S. Consiste en otorgar a la CPU un nivel de acceso completo.

b. El acceso a las direcciones de E/S se hace uso de Snap7 [32]; una suite de comunicaciones Ethernet multiplataforma de código abierto cuya API posibilita la interconexión con los PLC Siemens S7. En concreto se utilizan las funciones ilustradas en la Tabla 1.

Por otro lado, si el proyecto de automatización se ha realizado con Twincat, únicamente será necesario hacer uso de funciones facilitadas por ADS (Automation Device Specification) [33]. La Tabla 1 muestra las funciones necesarias.

Tabla 1: listado de funciones para lectura y escritura en PLCs

\begin{tabular}{|c|c|}
\hline \multicolumn{2}{|l|}{ Snap 7} \\
\hline Función & Propósito \\
\hline ConnectTo & $\begin{array}{l}\text { Conecta el objeto cliente } \mathrm{nl} \\
\text { PLC. }\end{array}$ \\
\hline SetConnectionType & $\begin{array}{l}\text { Establece el tipo de } \\
\text { conexión (PG/OP/S7Basic). }\end{array}$ \\
\hline SetConnectionParams & $\begin{array}{l}\text { Establece los parámetros de } \\
\text { la conexión. }\end{array}$ \\
\hline Disconnect & $\begin{array}{l}\text { Cierra la conexión con el } \\
\text { PLC. }\end{array}$ \\
\hline ReadArea & $\begin{array}{l}\text { Lee el área de memoria del } \\
\text { PLC indicado. }\end{array}$ \\
\hline WriteArea & $\begin{array}{l}\text { Escribe en el área de } \\
\text { memoria del PLC indicado. }\end{array}$ \\
\hline \multicolumn{2}{|l|}{ ADS } \\
\hline Función & Propósito \\
\hline adsSyncReadReq & $\begin{array}{l}\text { Lee datos de forma síncrona } \\
\text { del servidor ADS }\end{array}$ \\
\hline adsSyncWriteReq & $\begin{array}{l}\text { Escribe datos de forma } \\
\text { síncrona }\end{array}$ \\
\hline
\end{tabular}

\subsection{Integración y puesta en marcha}

La herramienta propuesta se basa en Processing, un lenguaje de programación multiplataforma y entorno de desarrollo integrado, visual y gráfico, de código abierto basado Java orientado al diseño gráfico [34]. Processing va dirigido a gente creativa, sin tener que ser necesariamente programadores informáticos. $\mathrm{Su}$ entorno es muy similar al de Arduino IDE y destaca por permitir programar la visualización de elementos como figuras geométricas, vectores, imágenes, videos, etc. 
Todo proyecto Processing (sketch) tiene dos funciones clásicas (setup y draw). La herramienta propuesta añade una tercera función (setting) encargada de interpretar los ficheros de configuración citados en apartados anteriores.

Las características y orden de ejecución de dichas funciones son:

1. void setting(): tiene como finalidad, la puesta en marcha del propio entorno Processing. Se ejecuta una sola vez y al comienzo del proyecto. Tras su ejecución, se conocen qué elementos/módulos forman la planta virtual.

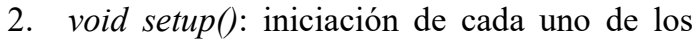
módulos que componen la planta virtual. Se ejecuta una sola vez.

3. void $\operatorname{draw}()$ : se ejecuta cíclicamente y la secuencia de ejecución fijada es la siguiente:

a. Consultar las salidas del controlador. Para lo cual se hará uso de las funciones indicadas en la Tabla 1.

b. Aplicar lógica de los módulos de la maqueta virtual.

c. Refrescar el gráfico de la maqueta virtual.

d. Actualizar las entradas del controlador. Nuevamente, se hará uso de las funciones indicadas en la Tabla 1.

Se ha desarrollado una librería de clases java para facilitar el uso de los elementos básicos: cilindros, botoneras, señales luminosas... Estas clases están dotadas de métodos para aplicar tanto la lógica del módulo como su visualización, es decir, pasos b y c de la secuencia que se sigue dentro de la función draw.

\section{CASO DE ESTUDIO}

La Figura 3 presenta una maqueta real, muy utilizada en las prácticas de asignaturas de Grado y Másteres en la Escuela Politécnica Superior de Ingeniería de Jaén de la UJA.

La asignatura de Automática Industrial se imparte en el segundo curso de todos los grados de Ingeniería Industrial de la UJA. Una de las prácticas realizada consiste en el presando de una pieza, para lo que se dispone de dos cilindros neumáticos (A y $\mathrm{B}$ ) pilotados con válvulas electroneumáticas $5 / 2$ una biestable y otra monoestable. El cilindro A (vertical) es el encargado de prensar la pieza, en tanto que el cilindro B (horizontal) tiene como objetivo expulsarla una vez que haya sido prensada. Inicialmente ambos cilindros han de encontrarse retraídos.

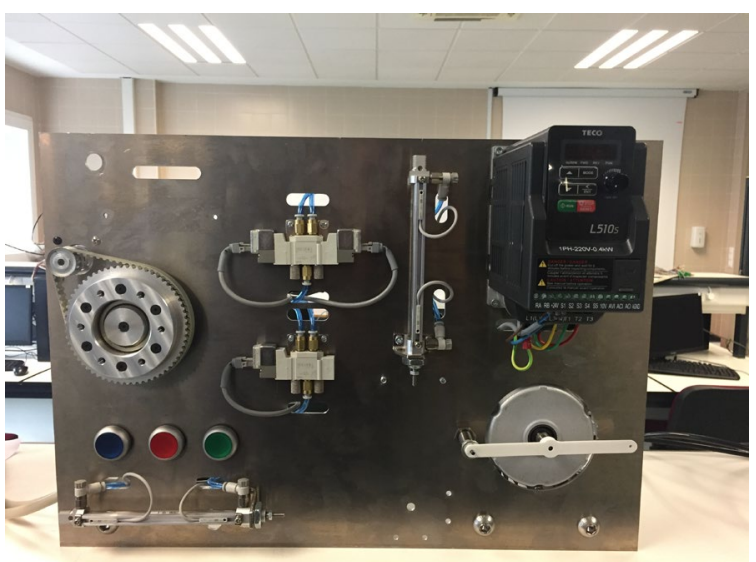

Figura 3: Maqueta disponible en laboratorios de la EPS

El operario tiene que colocar la pieza a prensar y posteriormente accionar el pulsador de marcha (PM). Una vez que el cilindro A se haya expandido, comienza la acción de prensado que tiene una duración de 6 segundos. Finalizado el prensado el cilindro A se contrae y se espera 2 segundos. Finalmente, se procede a expulsar la pieza para lo cual se ha de expandir el cilindro B durante 5 segundos, tras lo cual el cilindro se retrae y el automatismo queda listo para el siguiente prensado. Como se puede observar, los cilindros disponen de dos finales de carrera para saber si se encuentran contraídos o expandidos. El PLC utilizado para su automatización y control es S7 1200 de Siemens.

El curso académico 2020-2021 debido al COVID-19 el alumnado no pudo hacer uso de dicha maqueta. Y en su lugar se desarrolló la correspondiente maqueta virtual. La Figura 4 ilustra el fichero de configuración general, donde se hacen uso de dos cilindros y tres pulsadores, teniendo cada uno su correspondiente fichero de configuración (véase Figura 2.

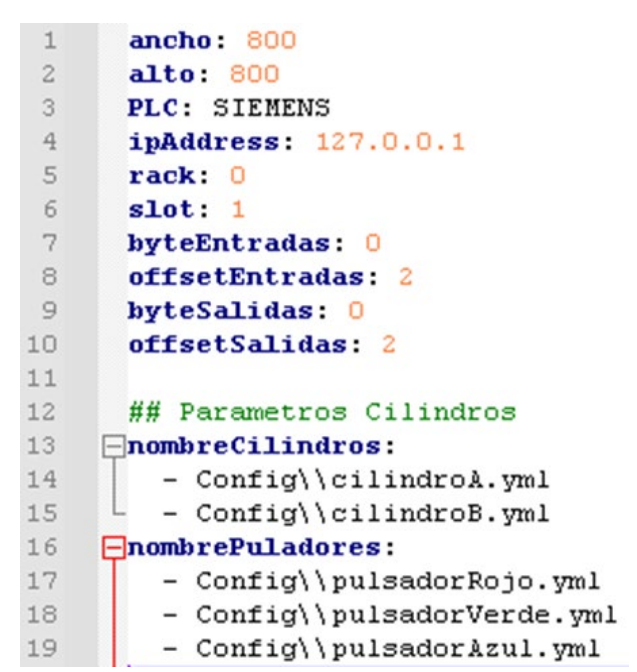

Figura 4: Fichero Configuración de maqueta de prensado 
El proyecto generado automáticamente que permite generar la virtualización de la maqueta ilustrada en la Figura 3 sigue las pautas ilustrados en el apartado anterior.

En concreto, la función draw de dicha planta virtual, generada automáticamente a partir del fichero de configuración es la ilustrada en

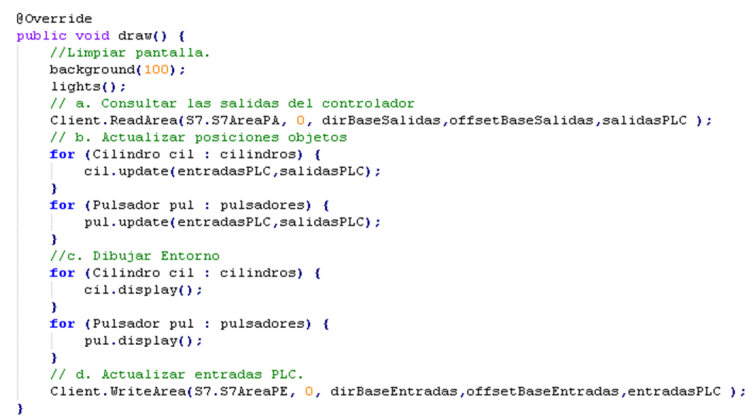

Figura 5: Función Draw para la maqueta virtual de prensado.

Finalmente, la maqueta virtual tiene la apariencia ilustrada en la Figura 6

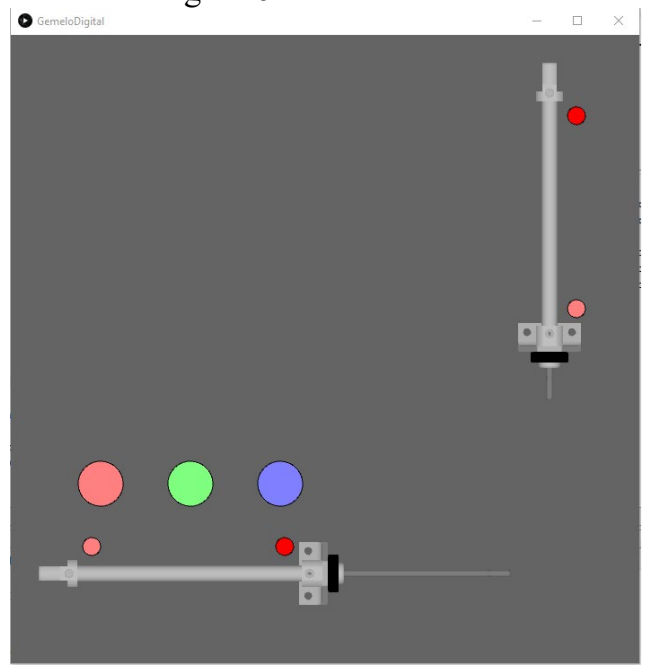

Figura 6: Maqueta virtual de prensado

\section{CONCLUSIONES}

El presente trabajo, se ha centrado en ofrecer unas pautas para la virtualización de maquetas de automatización. Aunque inicialmente tiene un carácter meramente docente, dicha aproximación también es válida para la definición de DT, entendido como modelo de simulación de alta precisión donde las E/S son las mismas que las de la planta real. La herramienta propuesta está basada en librerías de libre distribución y proporciona soporte para los fabricantes más extendidos en automatización, como son, Siemens y Beckhoff. Actualmente, las maquetas virtuales pueden estar formadas por elementos básicos como cilindro, sensores finales de carrera y pulsadores. En un futuro cercano se añadirán nuevas clases para manejar objetos como cintas transportadoras...

La herramienta propuesta procesa la información $\mathrm{del} / \mathrm{los}$ ficheros de configuración y genera automáticamente el código de proyecto Processing que da lugar a la maqueta virtual. Actualmente dicho(s) fichero(s) se generan a mano. Se está trabajando en ofrecer una interfaz gráfica que permita la generación automática del/los fichero(s) de configuración en formato yaml.

\section{Agradecimientos}

Los autores quieren agradecer la subvención parcial de este trabajo a través de los proyectos PIMED01_201921, PID2019-110291RB-I00.

\section{Referencias}

[1] S. Dormido, "Control learning: present and future," Annu. Rev. Control, vol. 28, no. 1, pp. 71-93, Jan. 2004.

[2] L. D. FEISEL and A. J. ROSA, "The Role of the Laboratory in Undergraduate Engineering Education,” J. Eng. Educ., pp. 121-130, 2005.

[3] S. R. Singer, M. L. Hilton, and H. A. Schweingruber, America's Lab Report. Washington, D.C.: National Academies Press, 2005.

[4] N. J. Nersessian, "Conceptual change in science and in science education," Synthese, vol. 80, no. 1, pp. 163-183, Jul. 1989.

[5] M. P. Clough, "Using the laboratory to enhance student learning," Learn. Sci. Sci. Learn., no. JANUARY 2002, pp. 85-94, 2002.

[6] J. E. Corter, J. V. Nickerson, S. K. Esche, C. Chassapis, S. Im, and J. Ma, "Constructing reality: A Study of Remote, Hands-On, and Simulated Laboratories," ACM Trans. Comput. Interact., vol. 14, no. 2, p. 7-es, Aug. 2007.

[7] J. E. Corter, J. V. Nickerson, S. K. Esche, and C. Chassapis, "Remote versus hands-on labs: a comparative study," in 34th Annual Frontiers in Education, 2004. FIE 2004., 2004, pp. 595-599.

[8] S. Chen, "The view of scientific inquiry conveyed by simulation-based virtual laboratories," Comput. Educ., vol. 55, no. 3, pp. 1123-1130, Nov. 2010.

[9] J. Ma and J. V. Nickerson, "Hands-on, simulated, and remote laboratories: A Comparative Literature Review," ACM Comput. Surv., vol. 38, no. 3, p. 7-es, Sep. 2006.

[10]H. Fry, S. Ketteridge, and S. Marshall, A Handbook for Teaching and Learning in Higher Education. 2009.

[11]I. E. Allen, J. Seaman, R. Poulin, and T. T. Straut, "Online report card: Tracking online education in the United States," 2016. 
[12]E. Commission, "ICT in Education," Digital Single Market. Digital Economy \& Society. [Online]. Available: https://ec.europa.eu/digitalsingle-market/en/ict-education.

[13] CRUE UNIVERSIDADES ESPAÑOLAS, "UNIVERSITIC 2015. Analisis de las TIC en las Universidades Españolas," 2015.

[14]D. Olin, J. Bourne, F. Mayadas, and S. Consortium, "Online engineering education: Learning anywhere, anytime.," J. Eng. Educ., no. January, 2005.

[15]I. Ruano Ruano, J. Gámez García, and J. Gómez Ortega, "Laboratorio Web SCORM de Control PID con Integración Avanzada," Rev. Iberoam. Automática e Informática Ind. RIAI, vol. 13, no. 4, pp. 472-483, Oct. 2016.

[16] J. Gómez-Ortega, I. Ruano-Ruano, J. GámezGarcía, J. R. Balsas-Almagro, and A. SánchezGarcía, "Laboratorio Remoto de un sistema de péndulo invertido sobre carro de trayectoria lineal.”Jaén, p. 105, 2014.

[17]B. Balamuralithara and P. C. Woods, "Virtual laboratories in engineering education: The simulation lab and remote lab," Comput. Appl. Eng. Educ., vol. 17, no. 1, pp. 108-118, Mar. 2009.

[18] C. Martin-Villalba, A. Urquia, and S. Dormido, "Development of an industrial boiler virtual-lab for control education using Modelica," Comput. Appl. Eng. Educ., vol. 21, no. 1, pp. 36-45, Mar. 2013.

[19] Dormido, R., Vargas, H., Duro, N., Sanchez, J., Dormido-Canto, S., Farias, G., \& Dormido, S.,2008. Development of a web-based control laboratory for automation technicians: The three-tank system. Education, IEEE Transactions on, 51(1), 35-44.

[20] Gómez-Estern, F., López-Martínez, M., \& de la Peña, D. M.,2010. Sistema de Evaluación Automática VíaWeb en Asignaturas Prácticas de Ingeniería. Revista Iberoamericana de Automática e Informática Industrial RIAI, 7(3), 111-119. DOI: 10.1016/S1697-7912(10)700479.

[21] UNILabs, Red de Laboratorios universitarios compartidos, 2021. Accesible online: https://unilabs.dia.uned.es/blog/index.php?entryi $\underline{\mathrm{d}=3}$

[22] García Aretio, Lorenzo (2021). COVID-19 y educación a distancia digital: preconfinamiento, confinamiento y posconfinamiento. Revista Iberoamericana de Educación a Distancia, 24(1), 9-25. Accesible online: https://www.redalyc.org/jatsRepo/3314/3314644 60001/331464460001.pdf

[23] Factory I/O, 2021. Accesible online: https://factoryio.com/

[24] FluidSim, Festo ES, 2021. Accesible online: https://www.festo.com/es/es/e/educacion/aprendi

zaje-digital/simulacion-virtual-y-modelacionid 31275/

[25] Gabor, T., Belzner, L., Kiermeier, M., Beck, M.T., Neitz, A., (2016) "A Simulation-Based Architecture for Smart Cyber-Physical Systems", 2016 IEEE International Conference on Autonomic Computing (ICAC), pp. 374-379.

[26] Grieves, M., (2015) Digital Twin: Manufacturing Excellence through Virtual Factory Replication.

[27] Vachálek, J., Bartalský, L., Rovný, O., Šišmišová, D., Morháč, M., Lokšík, M., Hoffmeister, M., Zimermann, P., (2017) "The digital twin of an industrial production line within the industry 4.0 concept", $201721 \mathrm{st}$ International Conference on Process Control (PC), pp. 258-262.

[28] Schroeder, G., Steinmetz, C., Pereira, C.E., Muller, I., García, N., Espindola, D., Rodrigues, R., (2019) "Visualising the digital twin using web services and augmented reality", 2016 IEEE 14th International Conference on Industrial Informatics (INDIN), pp 522-527.

[29] Platenius-Mohr, M., Malakuti, S., Grüner, S., Goldsch, T., (2019) "Interoperable Digital Twins in IIoT Systems by Transformation of Information Models: A Case Study with Asset Administration Shell", Proceedings of the 9th International Conference on the Internet of Things, pp 1-8.

[30] Weyer, S., Meyer, T., Ohmer, M., Gorecky, D., Zühlke, D., (2016) Future Modeling and Simulation of CPS-based Factories: an Example from the Automotive Industry, IFACPapersOnLine.

[31] yaml, 2021. Accesible online: https://yaml.org/

[32] Snap7, 2021. Accesible online: http://snap7.sourceforge.net/.

[33] ADS - Automation Device Specification) 2021, Accesible online: https://infosys.beckhoff.com/english.php?conten $\mathrm{t}=$../content/1033/cx8180 hw/5091854987.html \&id $=$.

[34] Processing, 2021. Accesible online: https://processing.org/ .

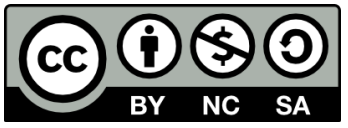
(C) 2021 by the authors. Submitted for possible open access publication under the terms and conditions of the Creative Commons Attribution CC BY-NC-SA 4.0 license (https://creativecommons.org/licenses/byncsa/4.0/deed.es). 